\title{
Evaluation of Delayed Fracture Characteristics of High-strength Bolt Steels by CSRT
}

\author{
Yukito HAGIHARA* \\ Department of Engineering and Applied Science, Faculty of Science and Technology, Sophia University, 7-1 Kioi-cho, Chiyoda-ku, \\ Tokyo, 102-8554 Japan. E-mail: hagihara@me.sophia.ac.jp
}

(Received on June 16, 2011; accepted on July 21, 2011)

\begin{abstract}
Delayed fracture is affected by two parameters: stress and hydrogen. Hydrogen enters the material from the environment as a result of corrosion. Generally, it takes a relatively long time, so that fracture occurs with a time lag after loading. The delayed fracture occurs when the driving force $(\mathrm{S}-\mathrm{H})_{\mathrm{E}}$ becomes greater than the material resistance $(\mathrm{S}-\mathrm{H})_{\mathrm{c}}$. Extensive research has been carried out to evaluate the material resistance against delayed fracture using the constant load test (CLT) and slow strain rate test (SSRT). Recently, the author has developed a test method called conventional strain rate test (CSRT). In CSRT, hydrogen, at a content corresponding to the accumulated hydrogen concentration in CLT and SSRT, is introduced uniformly into the specimen and loading is applied at a conventional strain rate with negligible hydrogen diffusion. The advantage of CSRT is that it requires no special testing equipment and the testing time is very much shorter than those of the previous test methods. The principle, test procedure and some experimental results of CSRT are discussed in this paper.
\end{abstract}

KEY WORDS: conventional strain rate test (CSRT); delayed fracture; high-strength steel; hydrogen embrittlement; SSRT; V-bearing steel; P-S-H evaluation.

\section{Introduction}

The demand for high-strength steels has recently been increasing in order to reduce the weight of structural components. High-strength steels are more susceptible to hydrogen-induced delayed fracture. ${ }^{1)}$ Therefore, it is important to evaluate the delayed fracture characteristics of high-strength steels quantitatively and accurately.

The delayed fracture characteristics are described on the basis of the critical combination of stress and hydrogen concentration at the fracture initiation site of the material. ${ }^{2-4)}$ The critical evaluation of delayed fracture based on hydrogen content $t^{5)}$ has been developed by introducing thermal desorption analysis (TDA), which enables the measurement of a small amount of hydrogen.

The delayed fracture of high-strength bolts with $1300 \mathrm{MPa}$ tensile strength (F13T) occurred in the 1960s, ${ }^{1)}$ and since then, extensive research on the mechanism of delayed fracture and evaluation of resistance to delayed fracture has been conducted. By simulating the actual condition of steel bolts, a constant load test (CLT) has been commonly used with a notched round bar (NRB) specimen. ${ }^{5,6)}$ The notch of the NRB specimen has a configuration and stress concentration similar to those of the screw of the bolt. In CLT, a constant load corresponding to the fastening stress of the bolt is applied and the critical hydrogen content is determined as a value in which delayed fracture does not occur for a certain period (100 or $200 \mathrm{~h}$ ). Delayed fracture of the bolt can be evaluated by comparing this critical value with the hydrogen content determined from the environmen- tal corrosive condition.

Since the critical hydrogen content determined by CLT is the material resistance to delayed fracture for a given notch geometry and applied load, CLT should be conducted when different notch geometries and loading conditions are considered. Then, the methods of evaluating the delayed fracture characteristics irrespective of the specimen configuration were studied..$^{2-4,7,8)}$ Wang et al. ${ }^{2-4)}$ have demonstrated by the slow strain rate test (SSRT) that the relationship between the maximum principal stress and instantaneously accumulated hydrogen concentration at the fracture initiation point during the test is independent of the notch geometry.

Both CLT and SSRT take a long time; thus, the specimen needs to be electroplated by cadmium coating after hydrogen precharging. ${ }^{6}$ On the other hand, the author ${ }^{9}$ has developed a convenient test method called conventional strain rate test (CSRT) of an NRB specimen. In CSRT, hydrogen, at a content corresponding to the accumulated hydrogen concentration in CLT and SSRT, is introduced uniformly into the specimen. The loading rate in CSRT is similar to that in the conventional tensile test, so that the hydrogen diffusion during the test can be neglected, and it takes only a few minutes to finish the delayed fracture test. By CSRT, many tests can easily be performed to evaluate the statistic and probabilistic delayed fracture resistance ${ }^{10)}$ and the delayed fracture characteristics of steels with strong hydrogen trapping sites such as V-bearing steel. ${ }^{11)}$ The present overview describes the principle and method of CSRT and the delayed fracture characteristics of various high-strength 
bolt steels determined by CSRT. ${ }^{9-11)}$

\section{Principle of CSRT}

It has been shown ${ }^{2-4,12,13)}$ that delayed fracture initiates slightly ahead of the notch of an NRB specimen, which nearly corresponds to the region where the maximum principal stress becomes maximum $\sigma_{\max }{ }^{*}$. Wang et al. $^{2-4)}$ have shown that the relationship between $\sigma_{\max }{ }^{*}$ and accumulated hydrogen concentration $H_{C} *$ at the fracture initiation point is independent of the notch geometry and becomes a material constant against delayed fracture, and is described using the following power law expression.

$$
\sigma_{\max } *=\alpha H_{C} * \beta
$$

Figure 1 shows the stress distribution in the NRB specimen with the geometry generally used (gross diameter $\mathrm{D}=10 \mathrm{~mm}$, notched portion diameter $\mathrm{d}=6 \mathrm{~mm}$ and notch tip radius $\mathrm{R}=0.1 \mathrm{~mm}$ of $\mathrm{V}$-notch). It is seen that the shape of the hydrostatic stress $\sigma_{m}$ distribution is similar to that of the principal stress $\sigma_{z}$ and the positions of the both maximum stresses coincide with each other. The accumulated maximum hydrogen concentration $H_{C} *$ is given by ${ }^{14)}$

$$
H_{C} *=H_{C} \exp \left(\Delta \sigma_{m} V_{H} / R T\right)
$$

where $H_{C}$ is the average hydrogen content in the specimen measured by TDA using a small sample with the fracture surface, $V_{H}$ is the change in volume produced in the lattice

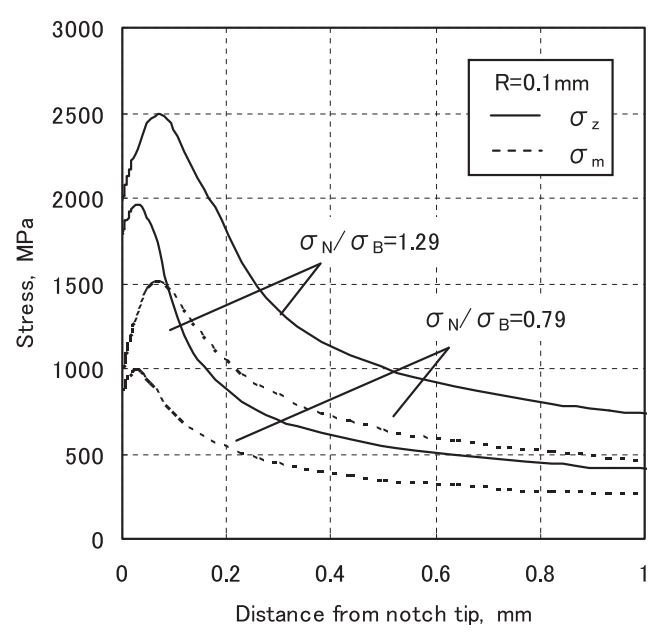

Fig. 1. Axial principal stress $\sigma_{z}$ and hydrostatic stress $\sigma_{\mathrm{m}}$ distribution ahead of the notch tip of NRB specimen. by the hydrogen $\left(V_{H}=2 \times 10^{-6} \mathrm{~m}^{3} / \mathrm{mol}\right), R$ is the gas constant $(R=8.314 \mathrm{~J} / \mathrm{mol})$ and $T$ is the absolute temperature. Moreover, $\Delta \sigma_{m}=\sigma_{m, \max }-\sigma_{m, \infty}$, where $\sigma_{m, \max }$ is the maximum hydrostatic stress and $\sigma_{m, \infty}$ is the hydrostatic stress away from the notched region. From stress analysis, a uniaxial stress field prevails at the unnotched region approximately $5 \mathrm{~mm}$ away from the notched line, so that $\sigma_{m, \infty}=\sigma_{z, \infty} / 3$.

In SSRT, as the hydrogen concentration is calculated using Eq. (2), it is necessary to provide sufficient time for hydrogen diffusion. Wang et al. ${ }^{15)}$ have shown that the cross -head speed should be less than $0.005 \mathrm{~mm} / \mathrm{min}$ to complete the diffusion for $\mathrm{Cr}-\mathrm{Mo}$ tempered martensitic steel. It takes at least several hours for SSRT. Thus, the reliability of $\mathrm{Cd}$ plating is key for SSRT to prevent hydrogen diffusion from the specimen during the test. Moreover, SSRT requires a special testing machine to control an extremely slow cross head speed. These are the disadvantages of SSRT.

Figures 2(a) and 2(b) illustrate instantaneous stress and hydrogen distribution in the specimen during CLT and SSRT, respectively. In CLT, the stress is applied under the precharged uniform hydrogen content and then, hydrogen diffusion and accumulation occurs. The hydrogen diffuses and accumulates at the stress-concentrated region according to Eq. (2). The time for complete diffusion is considered to be 100 or $200 \mathrm{~h}$ for the conventional NRB specimen of $\mathrm{D}=10 \mathrm{~mm}$. In SSRT, the applied stress increases gradually and the hydrogen diffuses according to the stress distribution simultaneously. In both tests, delayed fracture occurs when the accumulated hydrogen concentration satisfies the material resistance given by $\sigma_{\max } *-H_{C} *$.

In contrast, in CSRT, hydrogen, at a content corresponding to the accumulated hydrogen concentration of CLT or SSRT, is introduced uniformly into the specimen and then CSRT loading is started, as shown in Fig. 2(c). In CSRT, the loading speed is sufficiently high to ignore the hydrogen diffusion. When the stress increases to the level given by $\sigma_{\max }{ }^{*}-H_{C}{ }^{*}$, delayed fracture takes place. Normally, the cross head speed is $1 \mathrm{~mm} / \mathrm{min}$, which is approximately 200 times higher than that of SSRT, so that the testing time is a few minutes.

\section{Delayed Fracture Characteristics Determined by CSRT}

\subsection{Delayed Fracture Characteristics of $\mathrm{Cr}-\mathrm{Mo}$ High- Strength B13 and B15 Steels}

CSRT $^{9)}$ was carried out using specimens with different

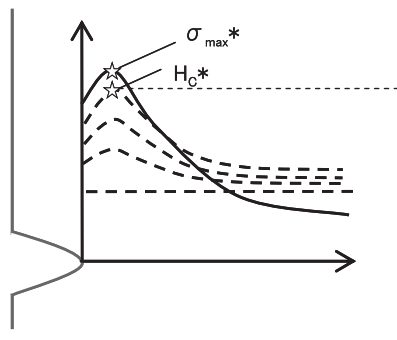

(a) $\mathrm{CLT}$

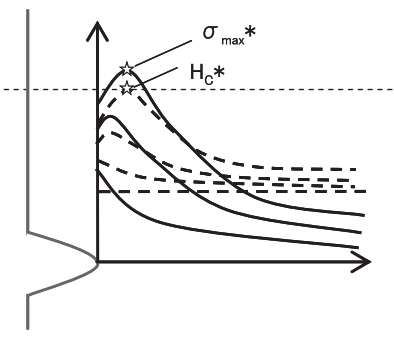

(b) SSRT

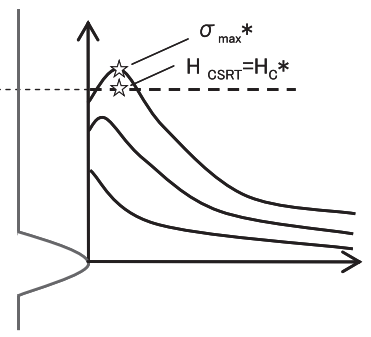

(c) CSRT

Fig. 2. Schematic illustration of principal stress and hydrogen concentration distribution depending on testing time or applied load, (a) CLT, (b) SSRT and (c) CSRT (放 indicates delayed fracture initiation site). 
notch tip radii on $\mathrm{Cr}-\mathrm{Mo}$ steel with a tensile strength of $1300 \mathrm{MPa}$ (B13-A). The maximum accumulated hydrogen concentration obtained by SSRT on similar steel was 4 mass ppm, which was 2-3 times higher than the average hydrogen content. ${ }^{2)}$ The hydrogen charging condition was studied at first to introduce more than 4 mass ppm uniformly into the specimen. ${ }^{9)}$ Hydrogen charging was performed by cathodic electrolysis for a sufficient time for hydrogen to absorb uniformly into the specimen. An aqueous solution of $3 \% \mathrm{NaCl}+0.3 \mathrm{~g} / \mathrm{L} \mathrm{NH}_{4} \mathrm{SCN}, 3 \% \mathrm{NaCl}+3 \mathrm{~g} / \mathrm{L} \mathrm{NH}_{4} \mathrm{SCN}$ or $0.1 \mathrm{~N} \mathrm{NaOH}$ was used to vary the hydrogen content. The temperature of the electrolyte was $30^{\circ} \mathrm{C}$ and the charging time was determined to be $72 \mathrm{~h}$ for the uniform absorption in the specimen with $\mathrm{D}=10 \mathrm{~mm}^{.9}$

During CSRT, the clip gauge displacement was measured between knife edges attached near the notch. Figure 3 shows the load-clip gauge displacement curves for the R0.1 specimen of B13-A as an example. ${ }^{9)}$ The fracture load decreases rapidly with increasing hydrogen content. In addition, it is very interesting that the displacement at fracture shifts and the amount of expansion is larger for the specimens with higher hydrogen content. As shown later, this is caused by increasing the area of the intergranular fracture at

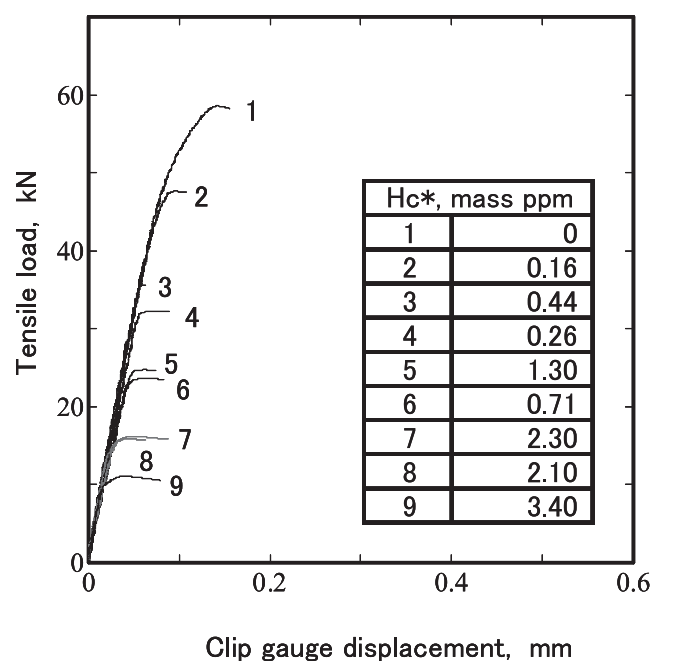

Fig. 3. Load-clip gauge displacement curves in CSRT for B13-A steel $(\mathrm{R}=0.1 \mathrm{~mm})$.

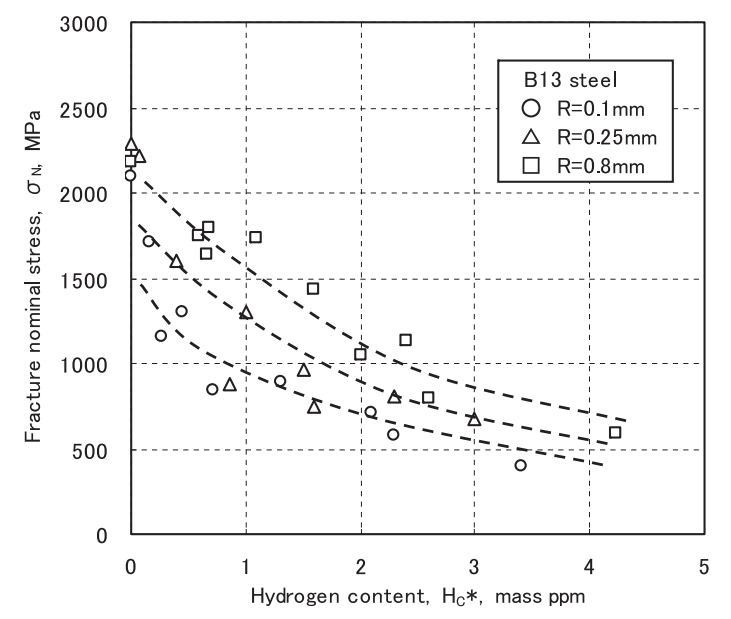

Fig. 4. Variation of fracture nominal stress $\sigma_{\mathrm{N}}$ with hydrogen content $H_{C} *$ obtained by CSRT for B13-A steel. fracture initiation sites.

Figure 4 shows the CSRT results with the relation $\sigma_{N}-$ $H_{C}{ }^{*}$, where $\sigma_{N}$ is the fracture nominal stress at the notched section. The hydrogen measured by TDA is the average hydrogen content $H_{C S R T}$ in the specimen, which is equal to the one at the fracture initiation point $H_{C}{ }^{*}$ in the CSRT specimen. The relation $\sigma_{N}-H_{C} *$ depends on the notch geometry and the fracture stress decreases with decreasing notch tip radius. When the results are analyzed using the maximum fracture stress, the relation $\sigma_{\max } *-H_{C} *$ is independent of the notch geometry as shown in Fig. 5 and expressed using Eq. (1). The $\sigma_{\max } *$ values were determined from the result of

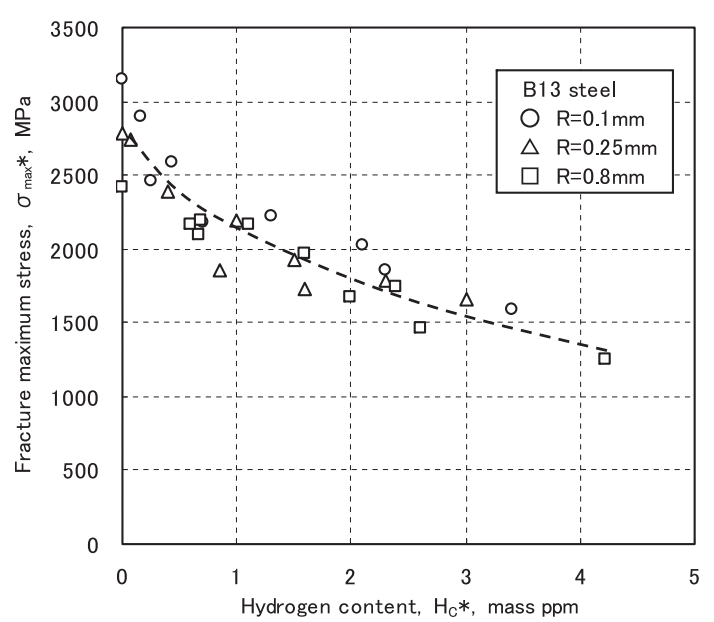

Fig. 5. Variation of fracture maximum stress $\sigma_{\max } *$ with hydrogen content $H_{C}{ }^{*}$ obtained by CSRT for B13-A steel.

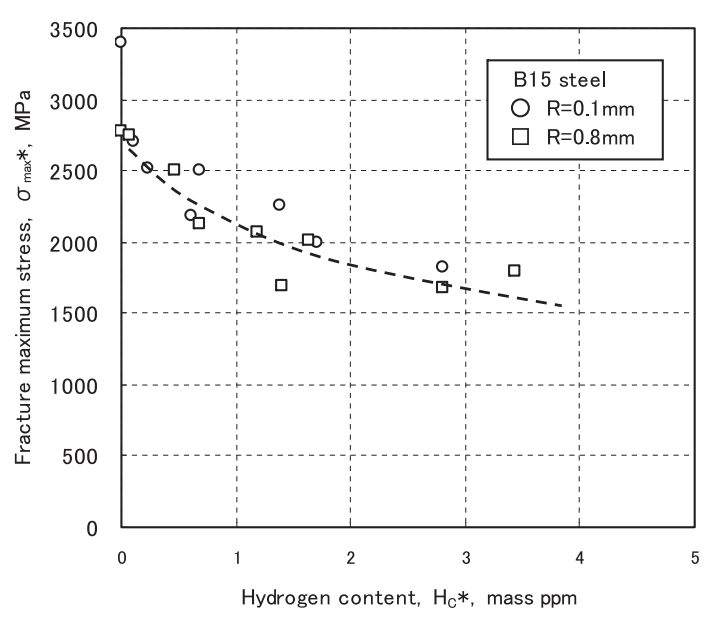

Fig. 6. Variation of fracture maximum stress $\sigma_{\max } *$ with hydrogen content $H_{C} *$ obtained by CSRT for B15 steel.

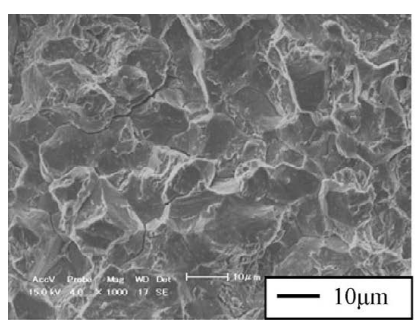

(a) $\mathrm{Hc}^{*}=2.3$ mass ppm

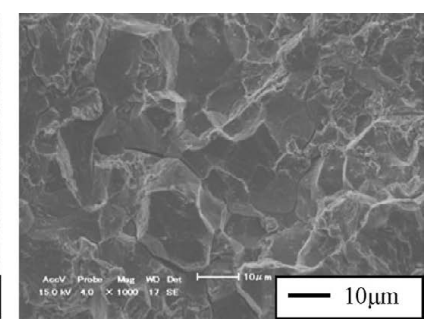

(b) $\mathrm{Hc}^{*}=0.26$ mass ppm
Fig. 7. SEM fractographs of B13-A steel with hydrogen contents of (a) 2.3 mass ppm and (b) 0.26 mass ppm. 
FEM stress analysis. A similar result was obtained on $1500 \mathrm{MPa}$ Cr-Mo steel (B15), ${ }^{9)}$ as shown in Fig. 6.

The fracture modes of B13-A and B15 steels at fracture initiation sites were intergranular (IG), as seen in Fig. 7. The fracture initiated ahead of the notch tip, which almost corresponds to the principal stress becoming maximum. Figure $\mathbf{8}$ shows the area of the IG fracture with various hydrogen contents. It is seen that the area of the IG fracture increases as the hydrogen content increases, which results in the fracture load decreasing. The trace of IG crack growth was observed in the load-clip gauge displacement curve shown in Fig. 3 as mentioned above.

\subsection{Delayed Fracture Characteristics of V-bearing Steel}

CSRT was applied to evaluate the delayed fracture characteristics of V-bearing steel with $1400 \mathrm{MPa}$ tensile strength (VC14). ${ }^{11)}$ The steel has finely dispersed vanadium carbide (VC), which acts as strong trap sites for hydrogen., ${ }^{6,20)}$ Therefore, it is very difficult to apply CLT and SSRT to this type of steel, because it takes a very long time for testing or needs an extremely low strain rate. On the other hand, CSRT can be applied, although the hydrogen precharging takes a much longer time than in the case of normal tempered martensitic steel such as B13. The hydrogen charging can be conducted using several specimens at once by preparing cathodic electrolysis equipment.

From a preliminary study on the hydrogen precharging condition, the time for the uniform absorption of hydrogen for $\mathrm{VC} 14$ steel was $120 \mathrm{~h}$ in the specimen of $\mathrm{D}=10 \mathrm{~mm} .{ }^{11}$ ) Figure 9 shows the variation of hydrogen content with

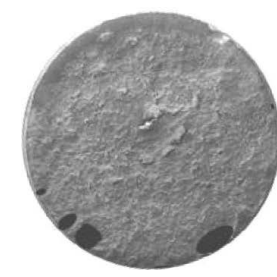

(a)

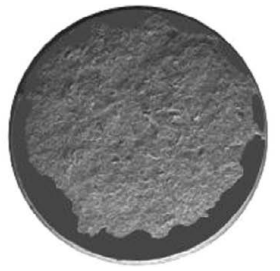

(b)

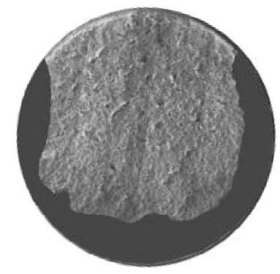

(c)
Fig. 8. Fracture surfaces indicating the area of intergranular fracture of B13-A steel with hydrogen contents of (a) 0.26 mass ppm, (b) 2.1 mass ppm and (c) 3.4 mass ppm.

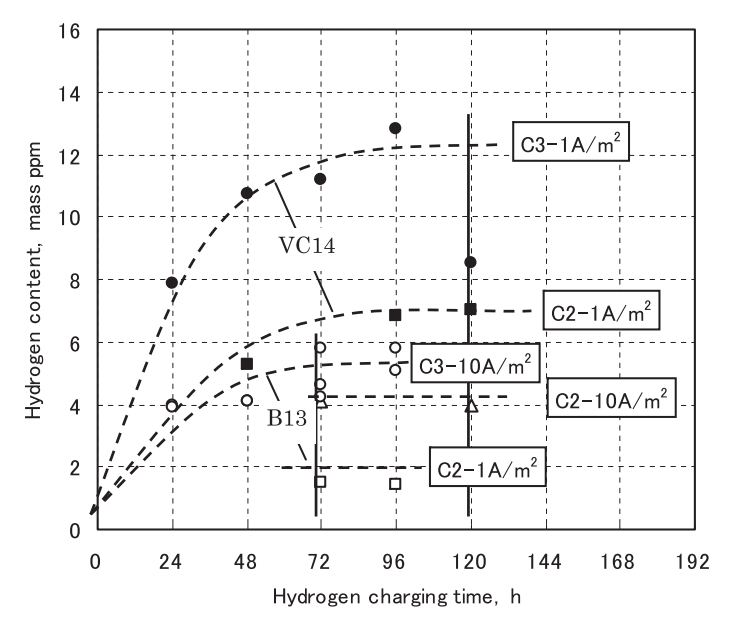

Fig. 9. Variation of hydrogen content with electrochemical hydrogen charging time for B13-A and VC14 steels. charging time for $\mathrm{VC} 14$ as compared with B13-A. The hydrogen content was much larger than that of $\mathrm{B} 13-\mathrm{A}$, which is caused by the trapping sites of VC particles. The CSRT results are shown in Fig. 10 with the relation $\sigma_{N}-$ $H_{C} *$ on VC14 steel. A kink was recognized in the $\sigma_{N}-H_{C} *$ curve. The fracture mode was quasi-cleavage (QC) for the specimen with a lower hydrogen content below the kink and IG for that with a higher hydrogen over the point. ${ }^{11)}$

\section{Statistical Aspects of Delayed Fracture Characteris- tics}

\subsection{P-S-H Analysis}

The time for CSRT is very short so that many tests can be easily preformed. Thus, the statistical analysis of delayed fracture data was conducted for B13-B steel. The delayed fracture resistance is described with the power law relation using Eq. (1). In the fatigue test, the statistical analysis called the P-S-N method was developed and standardized in JSME. $^{21)}$ In accordance with the P-S-N method, the probabilistic delayed fracture characteristics can be evaluated as denoted by P-S-H.

The CSRT results are plotted using the relation $\log \left(\sigma_{\max }{ }^{*}\right)-$ $\log \left(H_{C}{ }^{*}\right)$ as schematically shown in Fig. 11. The regression line for the data of $\left(x_{i}, y_{i}\right), i=1, n$ is expressed as follows.

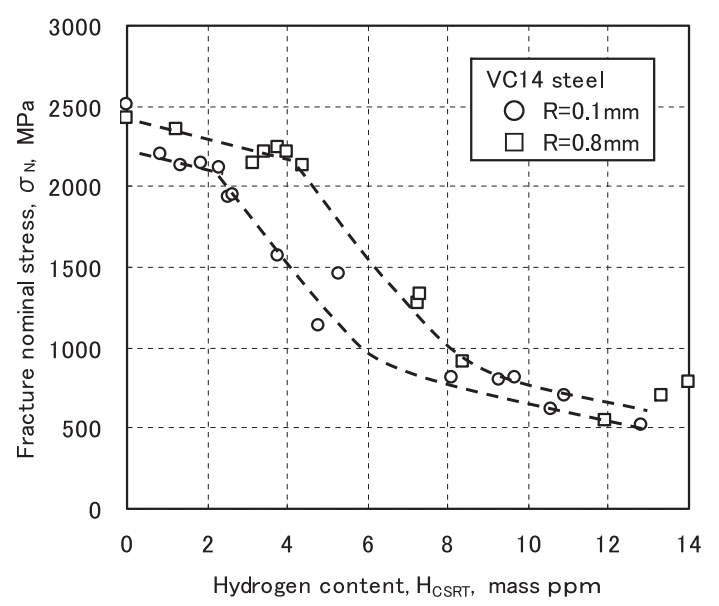

Fig. 10. Variation of fracture nominal stress $\sigma_{\mathrm{N}}$ with hydrogen content $H_{C}{ }^{*}$ obtained by CSRT for VC14 steel.

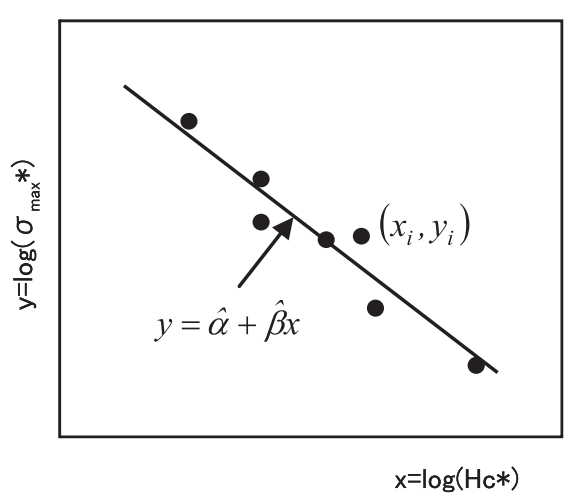

Fig. 11. Data of $\log \left(H_{C} *\right)-\log \left(\sigma_{\max } *\right)$ relation and regression line. 


$$
\begin{aligned}
& y=\hat{\alpha}+\hat{\beta} x \\
& \left.\begin{array}{l}
x=\log \left(H_{C} *\right) \\
y=\log \left(\sigma_{\max } *\right)
\end{array}\right\} \\
& \left.\begin{array}{l}
\hat{\alpha}=\bar{y}-\hat{\beta} \bar{x} \\
\hat{\beta}=\frac{n}{n-1} \frac{\operatorname{COV}\left(x_{i}, y_{i}\right)}{\operatorname{VAR}\left(x_{i}\right)}
\end{array}\right\}
\end{aligned}
$$

where, $\bar{x}$ and $\bar{y}$ are the average values of $x_{i}$ and $y_{i}$, respectively. By assuming that each data is normal-distributed around the regression line, the standard deviation is given by

$$
\hat{\sigma}=\left[\frac{1}{n-2}\left\{\left(y_{i}-\bar{y}\right)-\hat{\beta}\left(x_{i}-\bar{x}\right)\right\}^{2}\right]
$$

From Eqs. (3) and (6), the P-S curve with the probability $P$ is expressed using the following equation.

$$
y=\hat{\alpha}-k \hat{\sigma}+\hat{\beta} x
$$

If a normal distribution is assumed, the value of $k$ is determined, such as $k=1.282$ for $P=10 \%$. Figure $12^{10)}$ shows the P-S-H results for B13-B steel with $P=10 \%$ and $P=$ $50 \%$. The line of $P=10 \%$ nearly corresponds to the lower bound of the data scatter.

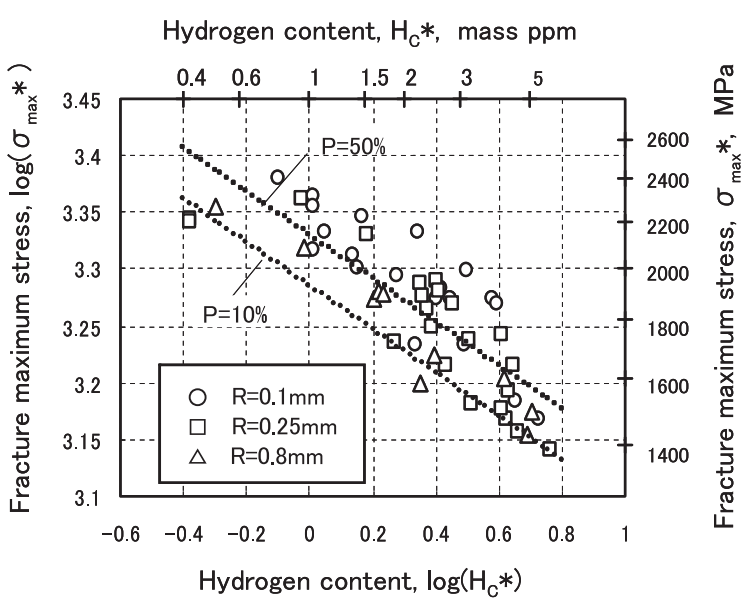

Fig. 12. $\mathrm{P}-\mathrm{S}-\mathrm{H}$ diagram of $\mathrm{P}=50 \%$ and $\mathrm{P}=10 \%$ for $\mathrm{B} 13-\mathrm{B}$ steel.

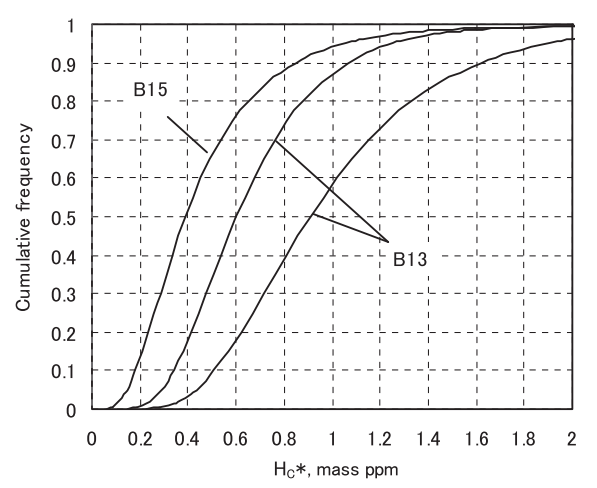

\subsection{Probabilistic Analysis of Delayed Fracture}

Steel bolts are used under a specified fastening condition. The critical hydrogen concentration $H_{C}{ }^{*}$ can be determined for that condition by statistical analysis. Corresponding to the fastening condition of steel bolts $y_{0}, x_{0 i}$ and the standard deviation $\hat{\sigma}\left(x_{0 i}\right)$ for each data are given by Eqs. (8) and (9), respectively, as shown in Fig. 13, and the probability density function (PDF) $f_{C}\left(x_{0 i}\right)$ and cumulative distribution function (CDF) $F_{C}\left(x_{0 i}\right)$ are expressed as Eqs. (10) and (11), respectively.

$$
\begin{aligned}
& x_{0 i}=x_{i}+\frac{\left(y_{0}-y_{i}\right)}{\hat{\beta}} \\
& \hat{\sigma}\left(x_{0 i}\right)=\left\{\frac{\sum\left(x_{o i}-x_{i}\right)^{2}}{n}\right\}^{1 / 2} \\
& f_{C}\left(x_{0 i}\right)=\frac{1}{\sqrt{2 \pi} \hat{\sigma}(x)} \exp \left\{-\frac{1}{2}\left(\frac{x-x_{0}}{\hat{\sigma}(x)}\right)^{2}\right\} \\
& F_{C}\left(x_{0 i}\right)=\int_{-\infty}^{x_{0 i}} f_{C}\left(x_{0 i}\right) d x
\end{aligned}
$$

In the case of the geometry of screw R0.25 and the fastening load of $\sigma_{N}=0.9 \sigma_{B}$,

$$
y_{0}=\log \left(\sigma_{\max } *\right)=\log \left(1.62 \sigma_{B}\right)
$$

From the CSRT results, the material constants in Eq. (7)

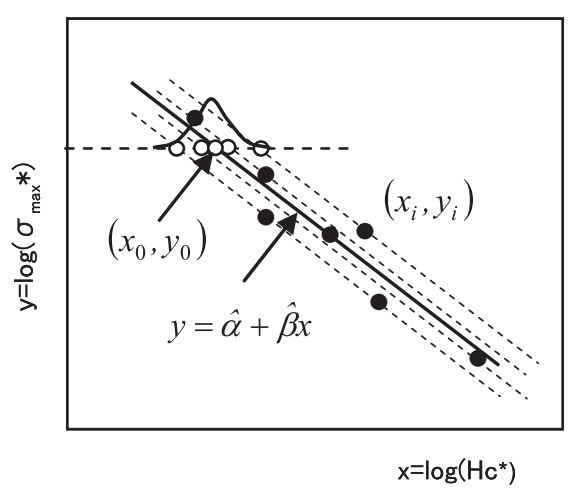

Fig. 13. Data scattering of $x_{i 0}$ for a given $y_{0}$ (normal distribution is assumed).

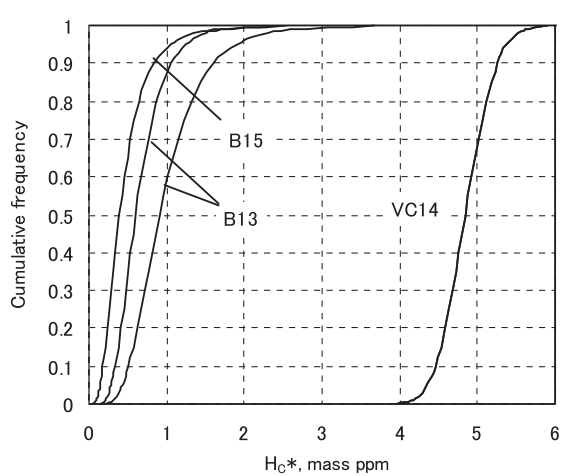

Fig. 14. Cumulative distribution of $H_{C} *$ for various bolt steels (B15, B13-A, B and VC14). 
are determined for B13-A, B, B15 and VC14 steels and the CDFs are given as shown in Fig. 14. It is seen that the critical hydrogen concentration $H_{C}{ }^{*}$ of B13-A, B is larger than that of B15 and the $\mathrm{VC}$ steel has a much higher $H_{C}{ }^{*}$.

The delayed fracture probability of steel bolts $P_{f}$ is expressed as the following equation, if the PDF $f_{E}(x)$ of hydrogen entry from the environment $H_{E}{ }^{*}$ is statistically independent of $H_{C}{ }^{*}$.

$$
P_{f}=\int_{0}^{\infty} f_{E}(x)\left\{\int_{0}^{x} f_{C}(\xi) d \xi\right\} d x=\int_{0}^{\infty} f_{E}(x) F_{C}(x) d x
$$

When $f_{E}(x)$ is assumed to be deterministic, Eq. (12) becomes $P_{f}=F_{C}(x)$. It has been demonstrated that the fracture probability of steel bolts for exposure tests can be evaluated by statistical analysis using $F_{C}\left(x_{0 i}\right)$ and the assumed environmental condition. ${ }^{22)}$

\section{Conclusions}

CSRT to evaluate the delayed fracture resistance has been proposed. The principle of CSRT is to introduce hydrogen at a content corresponding to the accumulated hydrogen concentration of CLT or SSRT $H_{C} *$ uniformly into the specimen. Then, loading at a conventional speed is carried out, which can neglect the hydrogen diffusion during the test. The main conclusions are summarized as follows.

(1) The procedure and method of introducing $H_{C}{ }^{*}$ uniformly into the specimen were established for several highstrength bolt steels. The maximum values of $H^{*}$ are more than 4 mass ppm for B13 steel and 14mass ppm for VC14 steel.

(2) The relation $\sigma_{\max } *-H_{C} *$ for B13 and B15 steels obtained from CSRT was independent of the notch geometry of the NRB specimen and became a material constant as recognized in SSRT.

(3) The delayed fracture resistance for $\mathrm{VC} 14$ steel was obtained by CSRT. The time for hydrogen charging was
$120 \mathrm{~h}$, which was much longer than that for B13 and B15 steels.

(4) The method of statistical and probabilistic evaluation of delayed fracture was proposed on the basis of the P$\mathrm{S}-\mathrm{N}$ procedure of fatigue test. The $\mathrm{P}-\mathrm{S}-\mathrm{H}$ of delayed fracture was evaluated for B13 steel.

(5) The methods of evaluating PDF and CDF of $H_{C}{ }^{*}$ were proposed and CDFs of $H_{C} *$ for B13, B15 and VC14 steels were obtained under the postulated fastening condition of steel bolts. The results correspond well to the fracture probability in exposure tests.

\section{REFERENCES}

1) S. Matsuyama: Delayed Fracture, The Nikkan Kogyo Shimbun, Ltd., Tokyo, (1989), 25.

2) M. Wang, E. Akiyama and K. Tsuzaki: Mater. Sci. Eng. A, 398 (2005), 37

3) M. Wang, E. Akiyama and K. Tsuzaki: Corros. Sci., 49 (2007), 4081.

4) M. Wang, E. Akiyama and K. Tsuzaki: Scr. Mater., 52 (2005), 403.

5) N. Suzuki, N. Ishii, T. Miyagawa and H. Harada: Tetsu-to-Hagané, 79 (1992), 97.

6) S. Yamasaki and T. Takahashi: Tetsu-to-Hagané, 83 (1997), 454.

7) S. Takagi, T. Inoue, T. Hara, M. Hayakawa, K. Tsuzaki and T. Takahashi: Tetsu-to-Hagané, 86 (2000), 689.

8) S. Takagi, S. Terasaki, K. Tsuzaki, T. Inoue and F. Minami: Q. J. Jpn. Weld. Soc., 22 (2004), 125.

9) Y. Hagihara, C. Ito, N. Hisamori, H. Suzuki, K. Takai and E. Akiyama: Tetsu-to-Hagané, 94 (2008), 215.

10) Y. Hagihara, C. Ito, D. Kirikae, N. Hisamori, H. Suzuki and K. Takai: Tetsu-to-Hagané, 95 (2009), 489.

11) Y. Hagihara, T. Shobu, N. Hisamori, H. Suzuki, K. Takai and K. Hirai: Tetsu-to-Hagané, 97 (2011), 143.

12) S. Yamazaki and T. Takahashi: Tetsu-to-Hagané, 87 (1997), 460.

13) S. Takagi, T. Inoue, K. Tsuzaki and F. Minami: J. Jpn. Inst. Met., 65 (2001), 1073.

14) A. R. Troiano: Trans. ASM, 52 (1960), 54

15) M. Wang, E. Akiyama and K. Tsuzaki: Scr. Mater., 53 (2005), 713.

16) T. Kushida, H. Matsumoto, N. Kuratomi, T. Tsumura, F. Nakasato and T. Kudo: Tetsu-to-Hagané, 82 (1996), 297.

17) T. Tsuchida, T. Hara and K. Tsuzaki: Tetsu-to-Hagané, 88 (2002), 771.

18) T. Omura, T. Kushida, K. Miyata and Y. Komizo: Tetsu-to-Hagané, 90 (2004), 106.

19) H. Asahi, D. Hirakami and S. Yamasaki: ISIJ Int., 43 (2003), 527.

20) T. Yokota and T. Shiraga: ISIJ Int., 43 (2003), 534.

21) JSME S002-1994: Standard Method of Statistical Fatigue Testing (2nd Edition), The Japan Society of Mechanical Engineers, Tokyo.

22) Japanese Society of Steel Construction: Tech. Rep., 91 (2010), 120. 